\title{
Forecasting Rock Burst in Coal Mines Based on Neural Network
}

\author{
Taifa Zhang*, Yajiang Zhang and Lihua $\mathrm{Mu}$ \\ College of Science, Heilongjiang University of Science and Technology, Harbin, China
}

\begin{abstract}
In view of disasters caused by rock burst becoming more and more serious in coal mine production, three models are established for evaluation and prediction the rock burst risk based on artificial neural network. First, ten indicators are determined which have a larger influence on rock burst. Then two back propagation network models are trained using the original data and the processed data reduced by principal component analysis respectively. And a radial basis function network model is also established using reduced data. Finally, the performance of three different neural network models are analyzed and the best scheme is determined for rock burst prediction.
\end{abstract}

Keywords: Back propagation, neural network, principal component analysis, radial basis function, rock burst.

\section{INTRODUCTION}

Rock burst in the coal mine is a special expression form of mine pressure and it belongs to mine dynamic phenolmenon [1]. Rock burst is a sudden release of energy in the coal and rock which are deposited in the mine roadway and stope. The coal and rock are thrown into the roadway by the power and a strong noise is made at the same time. It will cause vibration or damage of coal and rock, damage of supports and equipment, loss of life and personal injury, destruction of the roadway or other big problems $[2,3]$. Rock burst can also incur other mine disasters, especially, gas, coal dust explosion, fire and flood, which will interfere with the ventilation system, or destroy the ground vibration and buildings. Therefore, rock burst is one of the major disasters in coal mine $[4,5]$.

Due to the complexity of coal seams, although many scholars at home and abroad have made significant progress in the understanding occurrence mechanism and monitoring method of rock burst, there are also some limitations $[6,7]$. At present, the methods of rock burst prediction mainly include earth sound monitoring, experience analogy analysis, electrical impulses prediction, drilling cuttings, microseism monitoring, infrared radiation prediction as well as the method of determination of moisture content. In some certain conditions, these methods can achieve good effect. But all the factors influencing the rock burst are not taken into account comprehensively. So when and where the rock burst occurs can not be made timely and quantitatively. And the risk indexes of rock burst are difficult to determine $[8,9]$.

The conditions of rock burst are complicated and the influence factors are diverse. It has been unable to establish an effective mechanism for the prediction of rock burst accurately. The emergence and development of neural network provide a way to solve it. $\mathrm{Mr} \mathrm{Wu}$ once simulated the measured data comprehensively using back propagation (BP) network to guide the actual mining [10]. But the current researches of rock burst based on neural network are sketchy and many problems need to be optimized [11]. Such as the input variables are much more in the construction of a network, and correlation is not analyzed between them, which makes the structure of network is too complex [12, 13].

In order to solve the above problems, two kinds of neural network models are established for prediction the dangers of rock burst. First, technological and geological factors influencing the rock burst are carefully analyzed in the mining, and they are quantified to obtain the training samples according to the actual situation. Then, a BP network is designed for identification and prediction of the test samples, but its precision is not high enough. Then the data are carried on the principal component analysis (PCA) and some main factors are chosen. At the same time, a radial basis function (RBF) network is also established. Finally, their forecasting results are analyzed and the appropriate method is determined for the prediction of rock burst.

Next, we describe the material and methodology used in more detail.

\section{MATERIAL AND METHODS}

\subsection{The Influencing Factors of Rock Burst}

Rock burst is caused by various factors, which has a close relationship with the mining depth, geological structure, mining pressure, mining technological conditions and working face roof management and so on [1]. In general, the influencing factors of rock burst can be divided into two broad categories, which are shown in Table $\mathbf{1}$.

\subsection{The Structures of Neural Network}

Currently, the most widely used neural network models include the BP network and RBF network. The related theory has proved that a three layer network containing a hidden layer can approximate any continuous function, and a 
Table 1. Influencing factors of rock burst.

\begin{tabular}{|c|l|}
\hline Categories & \multicolumn{1}{c|}{ Concrete Terms } \\
\hline \hline Geological factor & Coal thickness, burial depth, coal seam dip angle, tectonic conditions, obliquity variation, coal thickness variation, gas density \\
\hline Technical factor & Roof arrangement, pressure relief situation, sound of coal burst \\
\hline
\end{tabular}

Table 2. Part of samples.

\begin{tabular}{|c|c|c|c|c|c|c|c|c|c|c|c|}
\hline No. & $\begin{array}{c}\text { Coal } \\
\text { Thickness }\end{array}$ & $\begin{array}{l}\text { Dip } \\
\text { Angle }\end{array}$ & $\begin{array}{l}\text { Burial } \\
\text { Depth }\end{array}$ & $\begin{array}{c}\text { Tectonic } \\
\text { Condition }\end{array}$ & $\begin{array}{l}\text { Obliquity } \\
\text { Variation }\end{array}$ & $\begin{array}{l}\text { Coal Thickness } \\
\text { Variation }\end{array}$ & $\begin{array}{c}\text { Gas } \\
\text { Density }\end{array}$ & $\begin{array}{c}\text { Roof } \\
\text { Arrangement }\end{array}$ & $\begin{array}{l}\text { Pressure } \\
\text { Relief }\end{array}$ & $\begin{array}{l}\text { Sound of } \\
\text { Coal Burst }\end{array}$ & Type \\
\hline 1 & 1.8 & 59 & 542 & 1 & 1 & 3 & 0.25 & 0 & 0 & 1 & weak \\
\hline 2 & 1.1 & 27 & 490 & 0 & 1 & 0 & 0.64 & 2 & 2 & 1 & medium \\
\hline 3 & 2.9 & 55 & 855 & 3 & 1 & 3 & 0.08 & 1 & 1 & 2 & micro \\
\hline 6 & 1.6 & 62 & 307 & 3 & 1 & 2 & 1.00 & 0 & 0 & 2 & micro \\
\hline 7 & 1.4 & 44 & 560 & 0 & 1 & 0 & 0.09 & 3 & 3 & 0 & strong \\
\hline 8 & 2.6 & 48 & 752 & 2 & 1 & 2 & 0.48 & 1 & 1 & 1 & weak \\
\hline 9 & 1.8 & 62 & 283 & 3 & 1 & 3 & 1.0 & 0 & 0 & 2 & micro \\
\hline
\end{tabular}

network containing two hidden layers can approximate any functions. In general, the neural network has one hidden layer, and it has two hidden layers only for the design of the discontinuous functions.

The neural networks with three layers are used for prediction and the transition functions apply s-type functions (1) from the input layer to output layer in the paper.

$$
f(x)=\frac{1}{1+e^{-x}},
$$

The linear functions are used for transition functions from hidden layer to output layer.

Usually, the node's number of input layer and output layer nodes are determined by the realities of situation. We can see from Table 1 that rock burst indexes have a total of 10 items. So the number of input nodes is 10 in the neural network model. In the experiments, 200 groups of rock burst data are obtained from the real mine production, and Table $\mathbf{2}$ shows the part of the samples.

The output of the neural network can be either a numerical variable or a linguistic variable. In general, linguistic variables can be represented using 1 from $n$ values notation, $n-1$ values notation or binary value notation. The danger of rock burst is divided into four categories: micro burst, weak burst, medium burst and strong burst. The n-1 values notation is used for translating linguistic variable into numerical variable in the paper. 000 indicates micro burst, 001 indicates weak burst, 010 indicates medium burst and 100 indicates strong burst. In this way, the number of output nodes is 3 in the neural network model.

To determine the node number of hidden layer is the key of the neural network algorithm. As long as the number of nodes in the hidden layer is enough, it can approximate any continuous function at arbitrary precision for neural network with only one hidden layer. But it will also increase the training time and even cause the excessive anastomosis of the network, which reduces the generalization ability of the network.

Because the number of nodes is less in the input layer and output layer, so the empirical formula (2) is used for determination the number of nodes in the hidden layer.

$$
m=\sqrt{n+l}+\alpha \text {, }
$$

$n$ and $l$ are numbers of nodes in the input layer and output layer respectively and is a constant between $[1,10]$.

The number of nodes in the hidden layer is changed constantly during the training of network and the best number of hidden nodes is chosen by comparing the training and testing error, training steps and network structure, etc.

\section{EXPERIMENTS AND RESULTS}

After the structure of network is determined, the top 195 groups of rock burst data are used for training samples. The last 5 groups of data plus 5 groups selected randomly from the training samples are used for tesing samples. In order to compare the performances between different samples, the samples need to be standardized before learning. The samples are converted within the scope of $[-1,1]$ using the formula (3), where $n \max X=1, n \min X=-1$.

\subsection{BP Network Prediction}

The above analysis shows that the number of input nodes is 10 and the number of output nodes is 3 . By experience, the 
learning rate of network is 0.05 , the maximal permissible error is 0.0005 and the maximal number of training is 50000 . Through repeated testing, when and the number of nodes in hidden layer is 12, the prediction is the best. After 211 times training, the network has achieved precision for 195 groups of samples, and the error decline curve is shown in Fig. (1). The results of 10 groups of testing samples are shown in Table 4.

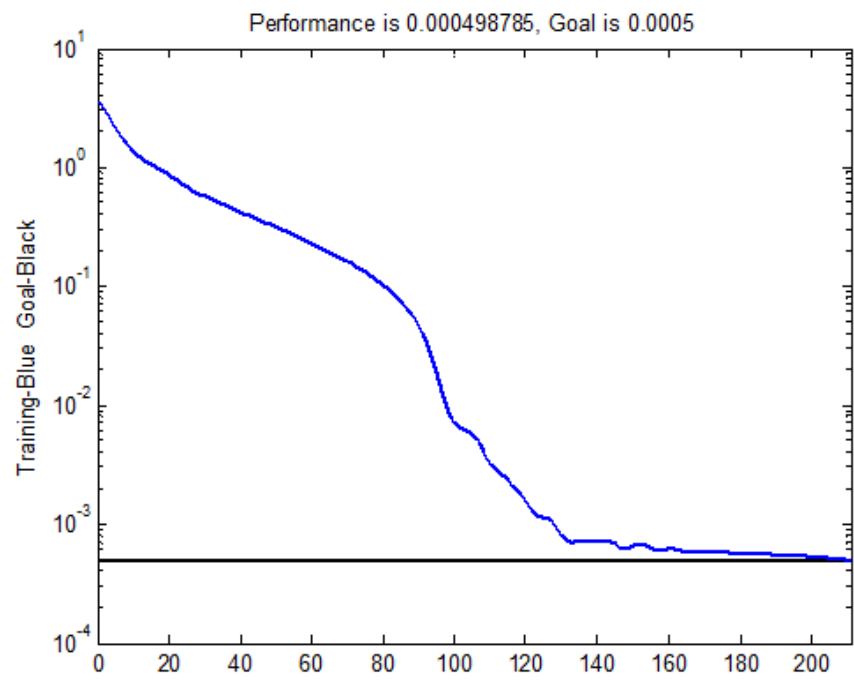

Fig. (1). The error decline curve of BP network.

\subsection{BP Networks Prediction Based on PCA}

The correlations have existed between different indexes of rock burst. Too many indexes can lead to excessive number of nodes in the input layer, which will make the structure of the network very complex and affect the precision of prediction. PCA is a common method to reduce the dimension of the data and it can reduce the degree of correlation between data.

The samples containing ten characteristics are analyzed using PCA. When the first four principal components are extracted, the accumulated variance has exceeded 80 percent of the total variance. The main factors are expressed in the formula (4). Substituting the standardized data into formula (4), four groups of principal data are obtained. Some reduced samples are shown in Table 3.

Now, the number of nodes in the input layer is 4 and the number of nodes in the output layer is 3 . The other parameters are the same as above network. Through repeated testing, when and the number of hidden nodes is 6 , the prediction is the best. After 812 times training, the network has achieved expected precision and the error decline curve is shown in Fig. (2). The results of 10 groups of testing samples are shown in Table 4.

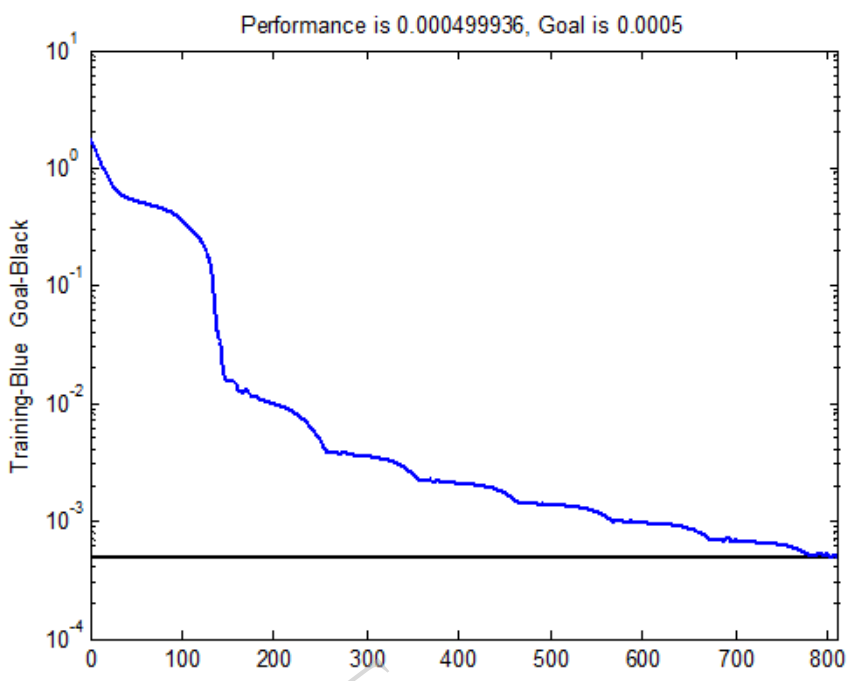

Fig. (2). The error decline curve of PCA based BP network.

\subsection{RBF Network Prediction Based on PCA}

The reduced samples are input to the RBF network with the same structure. The maximal permissible error is 0.0005 , the extending constant is 1 and the largest number of neurons is 20. The number of increased neurons between two demonstrations is 1 . The results of 10 groups of testing samples are shown in Table 4.

$$
\begin{aligned}
& x^{*}=\frac{(x-\min X)}{(\max X-\min X)}(n \max X-n \min X)+n \min X \\
& F_{1}=0.120 x_{1}+0.183 x_{2}-0.470 x_{3}+0.200 x_{4}+0.083 x_{5}+ \\
& 0.181 x_{6}+0.116 x_{7}-0.756 x_{8}-0.186 x_{9}+0.195 x_{10} \\
& F_{2}=0.160 x_{1}+0.029 x_{2}-0.420 x_{3}-0.064 x_{4}+0.436 x_{5}+ \\
& 0.190 x_{6}+0.353 x_{7}+0.424 x_{8}+0.542 x_{9}-0.146 x_{10} \\
& F_{3}=-0.447 x_{1}-0.033 x_{2}-0.508 x_{3}+0.148 x_{4}+0.288 x_{5}+ \\
& 0.249 x_{6}-0.358 x_{7}+0.111 x_{8}-0.065 x_{9}+0.163 x_{10} \\
& F_{4-}=0.441 x_{1}+0.221 x_{2}+0.675 x_{3}+0.205 x_{4}-0.318 x_{5}- \\
& 0.007 x_{6}+0.046 x_{7}-0.094 x_{8}+0.330 x_{9}-0.170 x_{10}
\end{aligned}
$$

The prediction accuracies of three networks are shown in Fig. (3). The BP network based on PCA has a smaller error and a higher prediction than the BP network obviously. For testing samples not trained, prediction accuracy of PCA

Table 3. Part of samples processed by PCA.

\begin{tabular}{|c|c|c|c|c|c|c|c|c|c|}
\hline No. & $\mathbf{F}_{\mathbf{1}}$ & $\mathbf{F}_{\mathbf{2}}$ & $\mathbf{F}_{\mathbf{3}}$ & $\mathbf{F}_{\mathbf{4}}$ & No. & $\mathbf{F}_{\mathbf{1}}$ & $\mathbf{F}_{\mathbf{2}}$ & $\mathbf{F}_{\mathbf{3}}$ & $\mathbf{F}_{\mathbf{4}}$ \\
\hline \hline 1 & 0.888 & -0.532 & 0.373 & 0.339 & 6 & 2.430 & -0.790 & -1.663 & 0.348 \\
\hline 2 & -2.439 & 0.517 & -0.889 & -0.582 & 7 & -3.485 & 0.847 & -0.036 & 0.882 \\
\hline 3 & 1.308 & 1.141 & 2.932 & 0.475 & 8 & 0.082 & 0.391 & 1.524 & 0.136 \\
\hline 4 & 0.555 & -0.196 & 1.104 & 0.534 & 9 & 2.783 & -0.547 & -1.667 & 0.356 \\
\hline 5 & -0.026 & -0.166 & 0.118 & 0.338 & 10 & -3.459 & 1.290 & -0.668 & 0.336 \\
\hline
\end{tabular}


Table 4. Testing results.

\begin{tabular}{|c|c|c|c|}
\hline \multirow{2}{*}{ No. } & BP Prediction & PCA Based BP Prediction & PCA Based RBF Prediction \\
\cline { 2 - 4 } & Error Sum Precision & Error Sum Precision & 0.0390 .961 \\
\hline \hline 1 & 0.0420 .958 & 0.0300 .970 & 0.0410 .959 \\
\hline 2 & 0.0390 .961 & 0.0330 .967 & 0.0370 .963 \\
\hline 3 & 0.0400 .960 & 0.0340 .966 & 0.0400 .960 \\
\hline 4 & 0.0390 .961 & 0.0310 .969 & 0.0380 .962 \\
\hline 5 & 0.0350 .965 & 0.0280 .972 & 0.0200 .980 \\
\hline 7 & 0.0270 .973 & 0.0240 .976 & 0.0140 .986 \\
\hline 8 & 0.0220 .978 & 0.0160 .984 & 0.0110 .989 \\
\hline 10 & 0.0200 .980 & 0.0110 .989 & 0.0080 .992 \\
\hline
\end{tabular}

based BP network is higher than PCA based RBF network obviously. And the results of PCA based RBF network are a little better than the PCA based BP network for testing samples trained. However, the stability of BP network is better relatively.

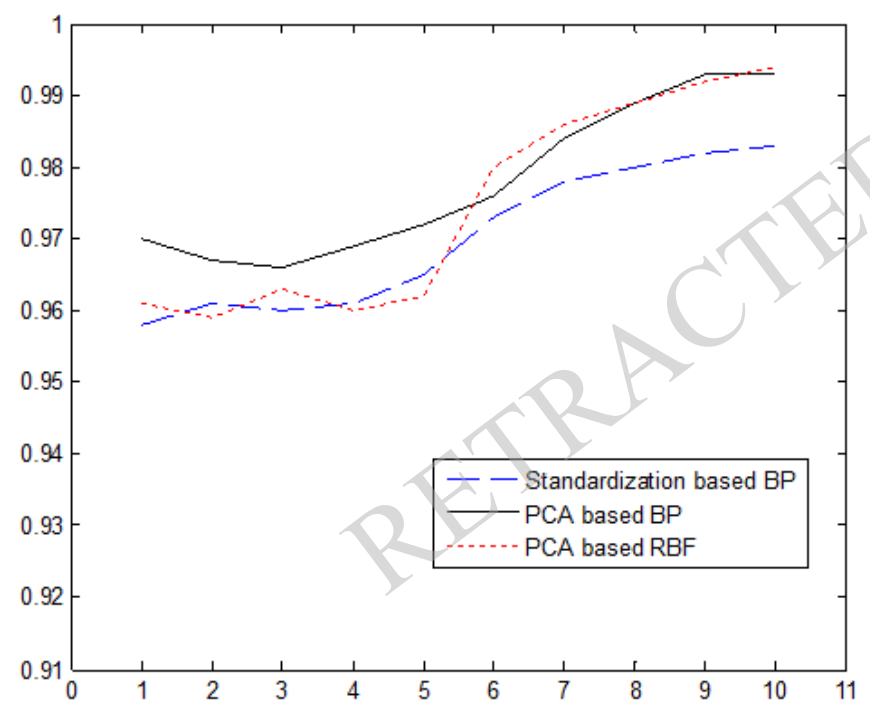

Fig. (3). Prediction accuracies of three networks.

\section{CONCLUSION}

In this paper, the neural network models are used for predicting the risk of rock burst and the PCA is utilized for reducing the original samples. Then the precisions of prediction are compared between original BP network, BP network based on PCA and RBF network based on PCA. The results of PCA based BP network is accordance with the real situation, so it can be used as the effective methods for predicting the rock burst risk.

Although the risk of rock burst in coal mine is successfully predicted in the paper, it still remains a lot to improve. Due to the limited samples, the network is trained inadequately. The selection of parameters has certain theoretical basis during the training of network, but the optimal parameters remains to be studied further.

We know of no previous register-based study that has illustrated the relevance of the original and PCA based models in an equally detailed manner as we have done here for prediction.

\section{CONFLICT OF INTEREST}

The authors confirm that this article content has no conflict of interest.

\section{ACKNOWLEDGEMENTS}

This work is supported by the Heilongjiang Natural Science Foundation (12531590) and scientific research project in College of Science, USTH (201305).

\section{REFERENCES}

[1] Minggao, Q.; Pingwu, S. Mining Pressure and Strata Control. China Mining University Press: China, 2003, pp. 54-78.

[2] Ghabonssi, J.; Sidasta, D.E.; Lade, P.V. Neural Network Based Modeling in Geomechanics Computer Methods and Advances in Geotechnics, Sirimavdance and Zaman USA, 2003, volume (1), pp. 23-37.

[3] Suo, Y. Research Based on the PSO-BP Neural Network to Forecast the Pressure from Working Face Roof in Shallow Seam, Master's thesis of Xi'an University of Science and Technology, 2010, pp. 13-46.

[4] Luochen, Z. Application of BP Neural Network in Different Lithology Identification Of Logging Interpretation, Master's thesis of Zhejiang University, 2011, pp. 5-12.

[5] Linming D.; Xueqiu, H. Technique of classification forecasting rock burst in coal mines. J. China Univ. Min. Technol., 2007, 36(6), 717-722.

[6] Lei, Z.; Fuhai, C. Introduction to the application neural network in mining engineering. Commercial Culture, 2008, 6, 172.

[7] Dubinski, J. Konopko, W. Tapnia-ocena, Prognoza, Zwalczanie. Glowny Instytut Gornictwa, Katowice, 2000, 20, pp. 1-20.

[8] Konopko, W. Doswiadczalne podstawy kwalifikowania wyrobisk gorniczych w kopalniach wegla kamiennego do stopm zagrozenia tapaniami. Katowice. Prace Naukowe w GIG, 1994, 795, 81-93.

[9] Qingxin, Q.; Yongxiu, W.; Debing, M.; Yi, L. Study on mechanism of rock burst induced by high strength mining with condition of non-hard roof. Chin. J. Rock Mech. Eng., 2005, 4(1), 5002-5006. 
[10] Yuliang, W. Study on Prediction Forecast of Rock Burst Based on Matlab Neural Networks. Master's thesis of Shandong University of Science and Technology, 2005, pp. 21-26.

[11] Xiaogang, F.; Chaohui, J. The cause analysis and countermeasures of coal burst at 5868 return air way heading face in Wulan coal mine. Colliery Saf., 2013, 44(10), 196-198.
[12] Zhaobo, C.; Yubin, L.; Jianchao, Z.; Hengting, L.; Zhongwei, L. Grey correlation analysis of human factors in ming accedents. Coal Eng., 2015, 47(4), 145-148.

[13] Quanlong L.; Xinchun, L. Effective stability control research of evolutionary game in China's coal mine safety supervision. $J$. Beijing Inst. Technol., 2015, 17(4), 49-56.

(C) Zhang et al.; Licensee Bentham Open.

This is an open access article licensed under the terms of the Creative Commons Attribution Non-Commercial License (http://creativecommons.org/licenses/ by-nc/3.0/) which permits unrestricted, non-commercial use, distribution and reproduction in any medium, provided the work is properly cited. 